\title{
Multiple Gene Transformation- Implications in Plant Breeding
}

\author{
Arshpreet Kaur Dhanoa*, Rahul Kapoor and Ritika Batra \\ Department of Plant Breeding and Genetics, \\ Punjab Agricultural University, Ludhiana, 141004, India
}

*Corresponding author

\section{Keywords \\ Transformation, Polygenic, \\ Transgenic, Genes, Agrobacterium tumefaciens \\ Article Info \\ Accepted: \\ 12 April 2019 \\ Available Online: \\ 10 May 2019}

A B S T R A C T

Transformation is the process by which the genetic make-up of an organism is altered by the insertion of new gene into its genome. As most of the agronomic traits are polygenic in nature, so the integration of multiple genes is required to manipulate the complex, polygenic metabolic or regulatory pathways, while ensuring their stable inheritance and expression in succeeding generations. There are various methods for multiple gene transformation but co-transformation method has proved more efficient and transplastomic technology can be used whenever have an option. It has many applications in plant breeding such as enhancing nutritional value like $\beta$-carotene content in rice, canola, maize and potato, vitamin E content in Arabidopsis, canola and soybean, synthesis of PUFA in Arabidopsis, impart biotic and abiotic stress resistance. Also, MGT has applications in molecular pharming.

\section{Introduction}

Plant transformation started in the early 1980s with the first conclusive demonstration that the causative agent of crown gall disease, Agrobacterium tumefaciens, could be harnessed by researchers to introduce defined fragments of DNA into plant cells. Plant transformation refers to the introduction and integration of foreign DNA in plant cells and the consequent regeneration of transgenic plants. Single gene transformation is the transfer of single gene from one organism to the other organism. Commercial example is $B t$ cotton which involves the transfer of one gene that is cry $1 A c$ gene from bacterium Bacillus thuriengenesis to cotton plants. As most of the agronomic traits are polygenic in nature, so plant genetic engineering will require the manipulation of complex metabolic or regulatory pathways involving multiple genes (Francois et al., 2002). Redirecting complex biosynthetic pathways and modifying polygenic agronomic traits requires the integration of multiple transgenes into the plant genome, while ensuring their stable inheritance and expression in succeeding generations. Manipulation of secondary metabolisms in plants and the production of biologically or 
pharmaceutically important multimeric proteins in plants (Hiatt et al., 1989) also require the introduction and expression of multiple genes in plants. As in disease control in plants, transgene-stacking methods are required. Resistance against e.g. fungal pathogens can be achieved by expressing a single gene coding for a protein with antifungal activity.

A more durable antifungal resistance can be achieved by combined production of antifungal proteins with different modes of action (Honnee, 1999) which also requires the integration of multiple transgenes into the plant genome. So the transfer, stable integration and expression of multiple genes into the plant genome is known as multiple gene transformation.

Multiple gene transformation enables the manipulation of entire metabolic pathway

Expresses multimeric proteins or protein complexes

Study complex genetic control circuits and regulatory hierarchies.

There are various methods for the multigene transformation as listed below

\section{Crossing/iterative strategy}

In this, two plants are crossed to obtain progeny that consists of the traits of the two parents. In the case of transgenic plants, a first gene is introduced in one of the parents and a second gene in the other parent. Crossing both transgenic parental lines result in progeny of which 25\% (in case both parents are hemizygous for the transgenes) or all (in case both parents are homozygous for the transgenes) contain the two transgenes. For instance, Datta and co-workers in 2002 developed disease and pest resistance rice by crossing plants expressing the Xa21 gene (resistance to bacterial blight) with plants expressing both a Bt fusion gene and a chitinase gene (confers resistance to yellow stem borer and tolerance to sheath blight respectively).

\section{Advantages and limitations of iterative strategy}

This is technically simple technique and involves transfer of pollens from one parent to the other, but precautions should be taken to avoid the self pollination. The major drawback is the only applicable to sexually propagated crops and obtaining homozygous plants is difficult. Also, introduced transgenes are not linked and can integrate at random locus of plant genome. This means that they will segregate apart again in subsequent generations.

\section{Sequential transformation}

Sequential transformation, or repeated transformation or re-transformation, is defined as the repetitive insertion of transgenes into a plant. For example, Singlepreek and co-workers in 2003 introduced twogene glyoxalase pathway into tobacco that led to enhanced salinity tolerance.

\section{Advantages and limitations}

This method is also applicable to vegetatively propagated and does not lead to loss of desirable combination of existing traits due to recombination. But is relatively time consuming, labour intensive, requires one selectable marker for every transgene and can induce gene silencing.

\section{Co-transformation}

It is the simultaneous introduction of multiple genes in a cell followed by the integration of genes in cell genome. Genes are either present on the same plasmid that is single plasmid cotransformation of linked genes or on separate 
plasmids that is multiple plasmid cotransformations of unlinked genes. Single plasmid co-transformation is the robust strategy for small number of input genes, but as the number increases, the vectors become unstable. In this, the genes to be introduced are linked as a single piece of DNA, with each gene having its own promoter and terminator. This method has advantage over the other methods that the integration of linked genes will take place on a single locus. So, the transgenes will be inherited stably to the next generations.

Other Multiple plasmids co-transformation involves several plasmids each carrying a different transgene. It has the advantage that assembly of the different expression cassettes is technically easier. The major limitation is that the T-DNA integration can occur at any chromosomal loci which will complicates further breeding.

Co-transformation is technically demanding, problem of gene silencing, difficulty to assemble complex plasmids with multiple gene cassettes and undesirable incorporation of complex T-DNA molecules from multiple sources.

\section{Transplastomic technology}

In this technology, Genes can be introduced into chloroplast genome via homologous recombination. As opposed to nuclear genes in plants, which transcribe singly, chloroplast genes are often present in operons.

First chloroplast transformation was done by Boynton and gillham in 1988 in alga Chlamydomonas reinhardti and in higher plants, first chloroplast transformation done in 1990 by Pal maliga and coworkers in tobacco.

Chloroplast transformation requires a robust method of DNA delivery into chloroplast, presence of active homologous recombination machinery in the plastid, and the availability of highly efficient selection and regeneration protocol.

\section{Advantages of chloroplast transformation Risk of transgene escape}

Chloroplast genome is maternally inherited and there is rare occurrence of pollen transmission. It provides a strong level of biological containment and thus reduces the escape of transgene from one cell to other.

\section{Expression level}

It exhibits higher level of transgene expression and thus higher level of protein production due to the presence of multiple copies of chloroplast transgenes per cell and remains unaffected by phenomenon such as pre or post-transcriptional silencing.

\section{Gene silencing/ RNA interference}

Gene silencing or RNA interference does not occur in genetically engineered chloroplasts.

\section{Position effect}

Absence of position effect due to lack of a compact chromatin structure and efficient transgene integration by homologous recombination. It avoids inadvertent inactivation of host gene by transgene integration.

\section{Disulphide bond formation}

Ability to form disulfide bonds and folding human proteins results in high-level production of biopharmaceuticals in plants.

\section{Homologous recombination}

Chloroplast transformation involves homologous recombination and is therefore 
precise and predictable. This minimizes the insertion of unnecessary DNA that accompanies in nuclear genome transformation. Also avoids the deletions and rearrangements of transgene DNA and host genome DNA at the site of insertion.

\section{Expression of edible vaccine}

High level of expression and engineering foreign genes without the use of antibiotic resistant genes makes this compartment ideal for the development of edible vaccines.

\section{Codon usage}

Chloroplast is originated from cyanobacteria through endosymbiosis. It shows significant similarities with the bacterial genome. Thus, any bacterial genome can be inserted in chloroplast genome.

\section{Expression of toxic proteins}

Foreign proteins observed to be toxic in the cytosol are non-toxic when accumulated within transgenic chloroplasts as they are compartmentalized inside chloroplast.

\section{Multiple gene expression}

Multiple transgene expression is possible due to polycistronic mRNA transcription.

\section{Challenges faced by chloroplast transformation}

- Applicable to limited plant species

- Unavailability of genome sequence

- It requires homologous flanking regions for recombination and insertion of genes

- Phenotypic alterations of transplastomic plants

- Gene expression in non green plastids
- Degradation of target gene product

- Low success rate of gene insertion into chloroplast genomes

$\begin{aligned} & \text { Applications } \\ & \text { transformation }\end{aligned}$
of multiple gene

\section{Enhancement of nutrition}

\section{Carotenoids}

There is 23 fold increase in carotene content in Rice (Ye et al., 2000), Canola (Fujisawa et al., 2009), Maize (Zhu et al., 2008) and Potato (Ravanella et al., 2003)

\section{Poly Unsaturated Fatty Acids (PUFAs)}

Humans are unable to synthesize long - chain polyunsaturated fatty acids (PUFAs) such as linoleic acid and $\alpha$-linolenic acid due to the lack of methyl-end desaturases but these are essential for humans. So must be obtained from their diet.

Some can be sourced from plants, but very long chain PUFAs (>C-20) are only present in fish and certain microbes, making them difficult to obtain. So it would make possible to obtain very long chain PUFAs by genetic engineering involving manipulation of whole pathway.

In Arabidopsis thaliana, three genes were introduced by sequential transformation that is Isochrysis galbana $\Delta 9$ elongase, Euglena gracilis $\Delta 8$ desaturase and Mortierella alpina $\Delta 5$ desaturase. It increased the EPA (eicosapentaenoic acid) content by 3\% and ARA (arachidonic acid) by $6.6 \%$ of total fatty acids (Qi et al., 2004).

\section{Vitamin $\mathbf{E}$ content}

There is $25 \%$ increase in vitamin $\mathrm{E}$ content of Indian mustard (Wu et al., 2005) and 20\% 
increase in soybean (Truksa et al., 2005) using Agrobacterium co-transformation of linked genes

\section{Impart resistance to biotic and abiotic stresses}

Multiple resistance in rice against bacterial blight, yellow stem borer and sheath blight (Datta et al., 2002).

Introduction of two-gene glyoxalase pathway into tobacco lead to salinity tolerance (Singlapareek et al., 2003).

\section{Development of super nutritious maize}

In maize, the level of 3 vitamins that is $\beta$ carotene, ascorbate and folate was increased specifically in the endosperm through simultaneous modification of 3 separate metabolic pathways. The transgenic kernals were found to contain 169-fold the normal amount of $\beta$-carotene, 6-fold the normal amount of ascorbate and double the normal amount of folate (Naqvi et al., 2009).

\section{Molecular farming/ Pharming}

High yield production of biopharmaceuticals like Vaccines, Antibodies and Next generation antibiotics (Lopez et al., 2013).

\section{References}

Datta, K., Baisakh, N., Thet, K. M., Tu, J. and Datta, S. K., 2002. Pyramiding transgenes for multiple resistance in rice against bacterial blight, yellow stem borer and sheath blight. Theor. Appl. Genet. 106, 1-8.

Francois, I. E. J. A., Willem, F. B. and Cammue, B. P. A., 2002. Different approaches for multi-transgene-stacking in plants. Plant. Sci. 163, 281-295.

Fujisawa, M., Takita, E., Harada, H., Sakurai,
N., Suzuki, H., Ohyama, K., Shibata, D. and Misawa, N., 2009. Pathway engineering of Brassica napus seeds using multiple key enzyme genes involved in ketocarotenoid formation. $\mathrm{J}$. Exp. Bot. 60(4), 1319-1332.

Hiatt, A. C., Cafferkey, R. and Bowdish, K., 1989. Production of antibodies in transgenic plants. Nature. 342, 76-78.

Honee, G., 1999. Engineered resistance against fungal plant pathogens. Eur. J. Plant. Pathol. 105, 319-326.

Lopez, U. Z., Masip, G., Arjo, G., Bai, C., Banakar, R., Bassie, L., Berman, J., Farre, G., Mirapleix, B., Massot, E. P., Sabalza, M., Sanahuja, G., Vamvaka, E., Twyman, R. M., Christau, P., Zhu, C. and Capell, T., 2013. Engineering metabolic pathways in plants by multigene transformation. Int. J. Dev. Biol. 57, 565-576.

Naqvi, S., Zhu, C., Farre, G., Ramessar, K., Bassie, L., Breitenbach, J., Conesa, D. P., Ros, G., Sandmann, G., Capell, T. and Christou, P., 2009 Transgenic multivitamin corn through biofortification of endosperm with three vitamins representing three distinct metabolic pathways. Proc. Natl. Acad. Sci. 106, 7762-7767.

Qi, B., Fraser, T., Mugford, S., Dobson, G., Sayanova, O., Butler, J., Napier, J. A., Stobart, A. K. and Lazarus, C. M., 2004., Production of very long chain polyunsaturated omega-3 and omega- 6 fatty acids in plants. Nat. Biotech. 22, 739-745.

Ravanello, M. P., Ke, D., Alvarez, J., Huang, B. and Shewmaker, C. K., 2003. Coordinate expression of multiple bacterial carotenoid genes in canola leading to altered carotenoid production. Metab. Eng. 5(4), 255-263.

Single-preek, S. L., Reddy, M. K. and Sopory, S. K., 2003. Genetic engineering of the glyoxalase pathway in tobacco leads to 
enhanced salinity tolerance. Proc. Natl. Acad. Sci. USA 100, 14672-14677.

Truksa, M., Wu, G., Vrinten, P. and Qiu, X., 2005 Metabolic engineering of plants to produce very long-chain polyunsaturated fatty acids. Transgenic. Res. 15, 131-137.

Wu, G., Truksa, M., Dalta, N., Patricia, V., Bauer, J., Zank, T., Cirpus, P., Heinz, E. and Qiu, X., 2005. Stepwise engineering to produce high yields of very long-chain polyunsaturated fatty acids in plants. Nat. Biotech. 23, 10131017.
Ye, X., Al-babili, S., Kloti, A., Zhang, J., Lucca, P., Beyer, P. and Potrykus, I., 2000. Engineering the provitamin A (beta-carotene) biosynthetic pathway into (carotenoid-free) rice endosperm. Sci. 287, 303-305.

Zhu, C., Naqvi, S., Breitenbach, J., Sandmann, G., Christau, P. and Capell, T. 2008. Combinatorial genetic transformation generates a library of metabolic phenotypes for the carotenoid pathway in maize. Proc. Natl. Acad. Sci. 105(47), 18232-18237.

\section{How to cite this article:}

Arshpreet Kaur Dhanoa, Rahul Kapoor and Ritika Batra. 2019. Multiple Gene TransformationImplications in Plant Breeding. Int.J.Curr.Microbiol.App.Sci. 8(05): 1437-1442. doi: https://doi.org/10.20546/ijcmas.2019.805.164 